\title{
ARTICLES
}

\section{Capacity Building and Capability Development: Understanding the Relations of Power and Exchange Between International Development Volunteers and Partner Organizations and Communities}

\author{
Khursheed Sadat
}

\section{Introduction}

The contributions of international development volunteers (IDVs) to the capacity development of partner organizations and communities has been well documented (Lough et al. 2011; Aked 2015; Lough/Oppenheim 2017; Nyirenda 2018; McLachlan/Binns 2019). Underexplored are the nature of these relationships, particularly as it concerns the relations of power within these exchanges between IDVs and partners, and their impacts on the process of capacity building. This paper fills this deficit by engaging in an analysis of the dynamics of power between these two parties and the ways in which it impacts the processes of capacity building of partner organizations and communities, bringing nuance to these discussions. In this paper, I draw upon the data from our study to confirm that IDVs contribute to the capacity building of partner organizations and communities through skills and capability development as well as being employed as resources. Furthermore, I argue that the relations of interaction and exchange between these two parties are constituted by complex and dynamic power relations in which both parties collaboratively shape how the capacity of organizations and communities are developed.

I begin the paper with a brief discussion of the capabilities approach as the theoretical lens around which this paper is framed. I follow this with a discussion of relevant scholarship, illustrating gaps in knowledge and situating this paper within it. Third, I include a detailed account of the findings as they relate to impacts of the engagements between IDVs and partner organizations on the process of capacity building. Finally, I analyze the findings of this research, deconstructing the ways in which interactions and partnerships between IDVs and partners shape the capacity building process. 


\section{Theoretical Framework and Literature Review}

The arguments and evidence of this paper are framed by the theoretical lens of the capabilities approach. Introduced by Sen (1999), this approach identifies development as constituting of building peoples' capability, which entails providing people opportunities and freedoms, particularly through access to healthcare and education, in order for them to be able to choose how to live (Sen 1999; Nussbaum 2011). Sen (1999) argues that this approach is agent-oriented as policies and practices of development are there to support people to shape their own lives and futures. Nussbaum (2011) makes the distinction between internal capabilities, trained or developed personal traits, and combined capabilities, the environment surrounding individuals that allows them to express their internal capabilities. In this paper I examine the influence of partnerships between IDVs and partner organizations on both types of capabilities. Specifically, in this paper I employ Nussbaum's adaptation of Sen's capabilities approach, which makes a conscious and explicit objective to address issues of gender inequality and injustice, as the main arguments and evidence on the process of capacity building center around power relations.

In line with the capabilities approach, I define capacity building of partner organizations and communities using the Organization for Economic Co-operation and Development (OECD) definition which refers to the central role of enabling environments in cultivating opportunities and freedoms for the growth and change of individuals and organizations (OECD 2011).

\subsection{Literature Review}

There is a wealth of literature examining the contributions of IDVs to the capacity building of host organizations (Lough et al. 2011; Nyirenda 2018; McLachlan/ Binns 2019). These contributions of IDVs consist of building organizational capacity through providing training to develop skills, such as in areas of documentation, computing and organizational management (McLachlan/Binns 2019; Nyrienda 2018). Research shows that IDVs have utilized their technical and professional skills to serve as resources to organizations, or serve as extra hands, a task accessible for even unskilled IDVs (Lough et al. 2011). Also, studies have revealed that IDVs have provided resources such as money and supplies, and even their own social capital to help sustain partner organizations (Lough et al. 2011). Furthermore, IDVs are found to enrich the intercultural competence of organizational staff through sharing knowledge and experiences (Lough et al. 2011). This body of literature on the contributions of IDVs to capacity building of partner organizations and communities generally situates these partners as recipients of services and resources, overlooking the practices of knowledge and skills transfer to the capability development of these partners, particularly as it relates to their expressions of agency in the process of building capacity, revealing scope for further research. 
Furthermore, these analyses of IDV contributions largely center on the instrumental value of these volunteers, necessitating discussions of the relationship of deeprooted structural issues, particularly gender inequality, to the process of capacity building, and whether and in what ways they are addressed in engagements between IDVs and partners.

In addition to highlighting the contributions of IDVs to the capacity building of partner organizations and communities, the literature also identifies several factors that pose challenges for IDV in this endeavor. The factors that mitigate the contributions of IDVs to capacity building include issues with language and communication, problematic attitudes of IDVs, issues of adaptation, issues of placement incompatibility as well as duration of service (Lough et al. 2011; McLachlan/Binns 2019; Nyirenda 2018; Tiessen/Lough 2018). The salient theme drawn from this list of mitigating factors is deficiencies in communication between hosts and IDVs, which suggests the need to further explore opportunities to enhance communication and nurture relationship-building.

Reflecting on the concept of relationality and its relevance to capacity building of host organizations, Lough et al. (2011) note that relationships between IDVs and organizational staff have the potential to be constituted by asymmetrical power relations, as the positionality of IDVs - who largely originate from "developed nations" - privileges their voice over local voices, which may result in the devaluation of local knowledge and cultures, an issue discussed in other scholarship (Perold et al. 2013; Hawkes 2014; Lough/Oppenheim 2017). This poses a challenge to capacity building as valuing local knowledge and skills are integral to the volunteer placements (Girgis 2007; Mclachlan/Binns 2019). The problematic role of outsider development actors who act as 'experts' on development issues in partner countries has been extensively covered in the development literature, with examples of superiority complexes and lack of mutual learning (Parpart 1995; Kothari 2005; Girgis 2007; Ferguson 2015). These studies provide scope for research on the relationships between/across IDVs, partner organizations, and communities in order to explore the potential for problematic and/or mutually beneficial relations of exchange and capacity building opportunities.

Development scholarship has explored the relevance of the quality of relationships between IDVs and host organizations as it relates to capacity building outcomes (Hawke 2014; Tiessen/Lough 2018; Mclachlan/Binns 2019). For instance, in a study of the factors that shape the contribution of IDVs to capacity building, Hawkes (2014) finds that the quality of the relationships between IDVs and hosts is an influential variable. Tiessen and Lough's (2018) research corroborates these findings, revealing that organizational staff perceive "team-oriented training" between IDVs and themselves as integral to practices of skills transfer, connecting it to experiences of greater ownership and sustainability of organizational 
capacity. Girgis (2007) witnesses that relationships of exchange between international development actors and local communities are successful at building capacity when development actors inquire into, respect and value local knowledge and skills, thus, characterizing partnership.

Relatedly, Impey and Overton (2013) argue that relationships based on partnerships are founded on reciprocity and respect. Aked (2015) expands on this idea and finds that partnerships are cultivated through informal practices in which parties connect on a personal level. Regarding reciprocity, Lough and Oppenheim's (2017) research offers insight into the multiple forms of reciprocal relationships between IDVs and hosts, documenting how these relationships are practiced, and the variables that shape the impact of these mutual relationships. These studies speak to the role of reciprocity in capacity building strategies, but offer limited details about the nature of the relationships, including the nuances of their dynamics, negotiation of power relations, strategies employed and opportunities for asserting agency among organizational staff and communities.

The findings analyzed in this paper offer insights into the distinctive contributions IDVs make through the process of relationship-building with partner organizations, highlighting the power dynamics of transnational relationships and opportunities for negotiation, mutual learning and reciprocal exchange.

\section{Methods}

Interviews were conducted in 2018 - 2019 with 150 partner organization staff in ten countries. Once all interviews were transcribed, data were coded to identify common themes throughout. Data were analyzed using discourse analysis. The introduction to this special edition has more detailed information on the methodology for data collection and the analysis of findings.

\section{Findings}

Findings from these data highlight many references to diverse strategies for capacity building and the impacts on capability development resulting from IDVs working in partnership with organizations around the world. The findings highlight several themes as they relate to capacity building, including knowledge and skills transfer; capability development; bridging the cultural divide; and IDVs as a vital resource for improving inclusion and participation. The data demonstrate the significance of partnerships, reciprocity, and relationship building as factors that shape the outcomes of IDV engagement with partner organizations and communities. In this section, I share the experiences of partner organizations who commented on their experiences working with IDVs and their insights regarding the impact of IDVs in relationship to capability development and capacity building. 


\subsection{Knowledge and Skills Transfer}

The findings reveal that international volunteers play an important role in bridging knowledge gaps of partner organizations and communities with regards to the process of capacity building. IDVs combine their expertise with intensive interactions with partner organization staff throughout their volunteer service and in so doing, also enhance their own capacities and skills-building through mutual learning in these transnational interactions.

Despite the two-way learning that is highly regarded as a major contribution of IDVs, there were many references to the nature of the skills and competencies that IDVs bring to partner organizations. Some of these skills included technical contributions such as creating and maintaining websites as well as online marketing and operating social media. The technical skills of documentation, which entails the specific procedure on how to write documents, particularly project reports, budget reports, as well as emails, were all identified across the research sites as necessary and valuable skills in operating the partner organizations. For instance, detailing the role of IDVs, one participant from Tanzania stated, "We do all things with her: documenting, analyzing, proposal [writing]; but also preparing the budget for the proposal." This participant illustrates the invaluable role IDVs play in supporting the partner organizational staff in operations of these partner organizations.

Other skills transferred through IDVs included time management, language and interpersonal skills. Particularly, the transfer of IDV language skills was reported by hosts as highly successful. For instance, one Nepalese staff member revealed, "I cannot speak proper English, but when volunteers are here I am responsible to communicate with them, so while communicating with them my English-speaking capacity had improved." Similarly, several partner organization staff members noted that their facility in English language improved as a result of IDV contributions.

The transfer of business management and entrepreneurial skills to host organizations and beneficiaries of the partner organizations was recognized as a salient outcome from IDV engagement with host organizations. For instance, the data on Malawi demonstrate that women were trained in dairy farming, which included tools and education on how to produce profit from such work. A participant from Ghana stated that through transferring entrepreneurial skills, IDVs supported the strengthening of women's economic voice, where farmers are equipped with the capability "to sell their produce and also negotiate for good prices." IDV support for women's economic voice was also evidenced in the data from Senegal, Uganda, Malawi, Guatemala and Tanzania, revealing that the transference of knowledge and skills on financial literacy and business development served to support women to become economically independent. 
The findings show that IDVs worked to strengthen analytical skills and helped develop these through engagement with hosts. For example, a participant from Tanzania speaking about the experience with an IDV reported how the IDV helped organization staff engage in critical analysis before making decisions and encouraged staff to look more deeply at the root causes of a problem. The IDV's contributions were considered highly valuable to the team, providing support to "be more analytical and unpack every kind of discussion which you have or any event which you encounter", which can help organization staff move beyond the symptoms of a problem and better understand the underlying reasons for development challenges. These critical analytical skills encouraged by IDVs were particularly important in relation to gender equality and women's empowerment (GEWE) work. As one study participant from Malawi noted: "I think we value what they have brought to the organization and contributions they make for us to have a better understanding on issues of gender and women empowerment. I think it's very beneficial.”

\subsection{Capability Development and Bridging the Cultural Divide}

The findings demonstrate that the transfer of situated cultural knowledge occurs through intercultural communication, which takes place through interactions between IDVs and hosts. For instance, the data from both Malawi and Vietnam reveal that cultural knowledge on Western working styles are shared, such as strict deadlines and clear boundaries between work and breaks, and when adopted they serve to promote efficiency within host organizations.

The findings reveal that cultural interactions between hosts and IDVs from diverse backgrounds work to promote diversity and the cultivation of intercultural and interpersonal skills. For example, a staff member of a partner organization in Peru reflecting on the diverse background of IDVs stated, "The fact that some of those people were immigrants [to Canada] has been very useful for us...because they bring different cultures and knowledge of the region that brings us a lot." This staff member recognizes the diverse backgrounds of Canadian IDVs as beneficial for their organization as they are gaining knowledge of the diverse cultures and experiences.

In relation to knowledge sharing related to GEWE, participants from Vietnam, Uganda and Peru highlighted the value of conversations between IDVs and hosts, including discussions on cultural differences in gender roles as important for shaping views and understandings of their own countries. For example, reflecting on their interactions with IDVs, a participant from Peru stated:

I like to converse about those things with them, because their culture has made it so they have more information on gender equality, and not only that they have more information, but they have been able to practice it and insert it in 
their lives and bring it to this country. It's great to be able to learn from them, so they don't only come to support us but they also teach us a lot on that theme. They have taught me a lot on feminism and equal opportunities.

This participant values the culturally specific knowledge on gender the IDV brought to their work, expressing how IDVs' knowledge shapes their daily practices as informed by feminism.

The findings from Kenya reveal that sharing situated cultural and experiential knowledge between IDVs and hosts introduces alternative realities, particularly to members of host communities, wherein their knowledge of the world expands as does their hope for their future opportunities. The findings from Senegal expand on this, identifying that both IDVs and hosts have their own knowledge and realities which do not always align. However, the interaction between the IDVs and partners facilitates the process of hybridization of knowledge and practices. A participant from Senegal asserts:

Well, now, it is best to take into account the values of one's own community and that of someone else and maybe, that is what allows us to formulate a new reality. That's how I see things but I don't think that certain things can directly influence what happens here.

This participant suggests that the residual effect of these interactions between IDVs and partners are gradual changes in organizational and community practices. In turn, these interactions with IDVs create opportunities to consider when and if change is needed to correct practices that are not working in the capacity building process for marginalized/oppressed people.

\subsection{Building Capabilities of IDVs}

Partner organization staff also provided a number of examples of ways that capacity building was carried out with the IDVs, highlighting the nature of exchange of knowledge and skill-sharing. For example, partner organizations identified the need to equip IDVs with knowledge of the context in which they are working in. For instance, one participant from Malawi suggested for IDVs "[t]o have a little bit understanding of where we are as a country at the moment, maybe to better understand the challenge that they will meet as we implement the project of gender [and] women['s] empowerment." Adding to this, a participant from Guatemala reinforced the importance of IDVs understanding "the local dynamics of the country where they are going to be, and to understand the cultural history and the limits that women have." Other important learning opportunities for IDVs included improved understanding of group dynamics and the nature of gender relations within the Guatemalan context, which are very specific to the cultural norms and practices of the country. The responsibility and ownership of partner organization 
staff for their organizations and communities is communicated in these suggestions and recommendations they provide IDVs, informing them what is required of them in their placement in order to work effectively. These examples highlight the contribution partner organizations make to expanding the knowledge of IDVs beyond the abstract or theoretical ideas they may bring with them. These exchanges between IDVs and partner organizations demonstrate the ways in which reciprocity and partnership are fundamental to the capacity building process.

\subsection{IDV as a Resource for Improved Inclusion and Participation}

The findings show that IDVs serve as resources in supporting inclusive participation and policy making with partner organizations and communities, a role that is highly valued by partner organization staff. Examples from partner organization staff in Peru, Uganda, Ghana, Malawi and Tanzania included their appreciation for the dedicated efforts of IDVs to champion gender equality as central to all work. IDVs engaged in a number of strategies to ensure a strong focus on gender equality including introducing a gender focus in the programs they operated; calling for increased participation of women in all activities to ensure equal representation; and supporting partner staff with the knowledge and tools to succeed in their roles. One example from Ghana highlights the championing role of IDVs in the training of broadcasters in gender and media. As one participant noted, IDVs:

Help to develop materials for the broadcasters, and also assist them with practical modules for gender inclusion in the local media. We had one that helped us to design a monitoring tool for capturing or measuring gender inclusion in our radio programming, and that was very fantastic (Ghana).

Moreover, in Malawi, IDVs advocated for increased participation of mothers in the workplace, working with host organizations to create a private space so working mothers can nurse their children at work, thus addressing the issue of equal participation in the public sphere.

For partner organization staff in Ghana, IDVs were identified as individuals who had a voice in issues of gender equality and were more likely to be heard than local staff when championing gender equality. The knowledge and experience that IDVs have with topics related to gender equality were welcomed by many who saw IDVs as better able to introduce new ideas. As one Ghanaian counterpart noted, IDVs have "distinctly impacted" their work, offering support in areas that can be off limits for some Ghanaians to address - "no-go" topics. The counterpart went on to say,

What I think they [international volunteers] have distinctly impacted is, you know our Ghanaian culture and the way some of these gender issues is a no-go area. I think it is easier for people to hear and accept it when it is coming from someone that is not a part of you. When you are a woman in our community, 
and you are talking about gender equality you look very strange to your male counterparts. But somebody from another culture bringing that helps (Ghana).

However, the findings from Uganda illustrate that sometimes the knowledge and voices of IDVs can be disregarded as irrelevant to the local context or seen as introducing foreign ideas that are not seen as applicable in the country. As a Ugandan study participant summarized,

Normally there are cultural issues within our society in terms of gender equality. I've seen international volunteers come up to speak about issues that people don't seem to consider of such importance. Often, this isn't taken very well and I don't think it's caused any issues within the organization.

Despite the reservations highlighted by this partner organization staff member, the study participant concluded the statement by indicating an appreciation for the contributions of IDVs, saying "I value their opinions", demonstrating the ambivalence and complexity surrounding the value of IDV contributions.

The findings from this study demonstrate that IDVs use their expertise to directly contribute to the operations of partner organizations. For instance, a participant from Uganda described the role of IDVs as supporting "our communications department in terms of developing articles, and especially updating our websites and updating our social media platforms." Adding to this, the data from Senegal reveals that IDVs help institutionalize partner organization efforts to improve the living conditions and political representation of Senegalese women by reaching out to women's groups, female politicians and community leaders by working to organize their pre-existing work structures and prepare official documents that could be presented to local political authorities and institutions. Thus, IDVs employ their skills with management, advocacy, documentation and social media to aid in the operations of organizations and support their work with international partners.

IDVs are valued for their contributions to communication and networking, such as facilitating communication between organizations and donors, development organizations, other local institutions, and the local communities. Examples of the interlocuter roles of IDVs were noted by participants in Malawi, Tanzania, Senegal, Uganda, Ghana, and Kenya. As the counterpart from Malawi noted, IDVs play an important role "as liaison person between us and the United Nations (UN), not UN as a whole but the United Nations Development Programme (UNDP)." Adding to this, the Malawian counterpart noted that the IDV is able to represent the organization in meetings.

A counterpart from Tanzania highlighted the important role of IDVs in facilitating networking and communications by working with ambassadors from different 
countries, building important connections to different ambassadors. In witnessing this contribution of IDVs, this Tanzanian participant went on to state, "now we are connected, because those offices now are calling, she's not here, but they are calling back." The impact of this networking role continues to be felt after the volunteer left and was considered essential for making their organization more visible and known to others - even more trusted within the community.

The study findings also provided insight into the valued services IDVs brought to gender equality work. As one study participant from Peru noted, the IDV brought expertise and experience that was "fundamental" to the team. The counterpart from Peru goes on to explain that the IDVs play an important role in the early stages of the gender network in Peru. The counterpart appreciated the diversity of professional IDVs that her organization worked with. One of the IDVs she worked with was a psychologist who "provided us with a different point of view to attend to the problems of violence against women or to analyze the situation of violence against women." The Peruvian counterpart concludes that the contributions of the IDVs were crucial to the success of the gender network. The expert skills of the IDV in this case were identified as fundamental to the operation of the organization.

\subsection{Factors That Facilitate the Collaborative Process}

At the heart of the contributions of IDVs to partner organizations and communities are a set of distinctive contributions and characteristics of the value addition of IDVs. Partner organizations explained these distinctive contributions as acts of reciprocity and dedicated partnerships cultivated through a commitment to relationship building.

Interactions between IDVs and partners are marked by the practice of reciprocity in which the knowledge and understanding of both sides are developed (Tanzania; Vietnam; Uganda; Malawi). For instance, a participant from Uganda explains:

Most of the volunteers, [...] before they go, we ask them to share their cultural backgrounds like: What happens in your culture?; How are women treated?; What are some of the gender roles that women are supposed to respect in their country? And then we have people here share what is happening in their communities. So when they share about ideas, they both learn from each other. The volunteers learn from the young girls here and they learn a lot from the volunteers.

Adding to this, a participant from Malawi said that they have learned so many things as a result of working with international volunteers but at the same time the Malawian partners "also equip them as well with how we do it here. So it's an exchange of experiences and innovative ideas." 
As the evidence demonstrates, the capacities of both parties are developed through interaction, constituting it as a collaborative engagement.

Similarly, experiences of partnership are observed when working with IDVs. One participant from Tanzania describes the partnership experience as working together, taking time to sit together and share what each considers is important for women. From these conversations, agreements on a way forward for supporting women is achieved, and a strategic plan is put in place for how to better reach women in their project activities and through trainings. The partnership is considered highly valuable in this case because it is a commitment to the exchange of ideas that shapes the next steps for completing the work. As partners in the co-design of strategies to address GEWE, both the partner organization and the IDV bring something to the table. It is their combined efforts and exchange of knowledge that is seen to advance the organization's work.

Another partner from Tanzania remarks that working with volunteers is beneficial because it adds value to the organization's existing work. Working alone can be challenging and the partnership model between partner organization staff and IDVs is generative, bringing up new and different ideas collectively. The process of bringing together individual views and ideas "contributes something as a whole, something new" (Tanzania). Thus, working in partnership allows for cultivating innovative solutions to issues, as occurs through the dialectical engagement between different parties who pool their intellectual and experiential resources.

Facilitating this collaborative process is the practice of building relationships. The findings illustrate that there are many factors that facilitate the process of relationship building between IDVs and partner organization staff. For example, a participant from Tanzania describes the nature of relationship building and the ways that this happens. For the Tanzanian counterpart, relationship building happens when "we sit together during lunch, we make friends, we talk. ... It's the chance we have to interact and share." As this testimony reveals, the practice of congregating in informal spaces to share experiences and knowledge, serves to cultivate friendships between IDVs and hosts. Similarly, the data on Senegal reveals that IDV live and work with the locals to cultivate connections and build relationships.

Reflecting on the value of relationships for knowledge and skills development, a participant from Guatemala states,

I think I value how she is as a person the most. Because listen, I could find someone with the best professional skills, but if we didn't connect at the personal level, then we wouldn't be able to accomplish the same things. So, this is how it is so much better that we have a close relationship. 
This final quote offers rich insights into the distinctive contributions that IDV programs make to relationship building and the nature of a reciprocal capacity building model. While capacity building research in IDV programs has largely focused on the transfer of skills or nature of competency building dynamics, these findings highlight the qualitative nature of how and when relationship building happens. These findings speak to the importance of building trust, mutual respect and friendship as characteristics of the partnerships that emerge between IDVs and organization staff in the Global South.

\section{Analysis}

The examples provided by partner organization staff of the role and contributions of IDVs to gender equality programming, skills development, and capacity building expands our understanding of the value accorded to IDVs by host organizations.

Beyond developing individual skills and general competencies, the findings summarized here demonstrate the nature of how relationships are formed in addition to sharing knowledge and skills. Together, these factors contribute to capability development for both the partner organization staff and the IDVs. The transfer of skills and knowledge between IDVs and partner organization staff and communities was found to be connected to experiences of agency and enhanced independence of partner organization staff. For example, the findings highlight how women in host communities worked with IDVs to gain knowledge of farming practice, as well as through training on business management and entrepreneurship. These services translated into new opportunities for women to generate an income and to advance their social and economic standing in their communities. Equipped with the skills and tools to manage themselves productively and independently, women were able to sell their produce and actively engage in determining the price of their products.

The development of individual capabilities noted in these examples and as defined by Sen's (1999) analytical lens, serves to expand people's freedoms. Capability development is recognized to take place particularly through practices of education, which aim to facilitate peoples increased participation in social, political and economic systems of their communities and beyond (Sen 1999). Previous studies exploring the relationship between gender and microfinance have discussed that participating in the public sphere and having entrepreneurial skills is not in itself empowering. The literature reveals a tension between a subject's agency and the structures of power surrounding them, namely patriarchy and capitalism, that limit agency (Roberts/Soederberg 2012; Wilson 2015).

The findings in this research project demonstrate that in addition to aiding the development of technical skills, IDVs engaged in the transfer of analytical skills 
with partner organizations and communities. Partners applied these analytical skills to theorize on the source and nature of the social, political and economic issues they are facing. For instance, as the findings from Tanzania reveal, discussions between both parties constituted a deeper inquiry into the root causes of issues serving to equip partner organizations with the tools to address problems and take a transformative approach, rather than simply addressing the symptoms of problems. Furthermore, the evidence from Malawi illustrates that through the practices of developing their analytical skills, partner organization staff reported that they were engaging in discussions of issues of gender inequality and women's empowerment with IDVs and found those experiences to have developed their understanding of these topics. These exchanges and discussions between IDVs and partner organizations and communities serve to build the capabilities of all parties involved, building their skill set while also transforming the environments in which they live and work. These exchanges are valued for addressing the structures that mitigate individual agency by constructing enabling environments that allow participants to be able to build their own capacities.

Attempts to address the source and nature of social, political and economic issues are evidenced to have also taken place through intercultural interactions which include discussions of lived experience between IDVs and partners. The findings reveal that both parties engaged in discussions of cultural traditions, practices and gender norms. McLachlan and Binns (2019) argue that cross-cultural exchanges constituted by relations of reciprocity serve to mitigate unequal power relations between IDVs and host communities as they both value the knowledge and experiences they share. Centering their analysis of the impacts of intercultural exchanges on the power dynamics between IDVs and host communities, these scholars leave unexplored a discussion of the impacts of the content of these exchanges on capability development (McLachlan/Binns 2019).

This study has demonstrated that sharing cultural knowledge provides partners with innovative tools to address problems and build for their future. For instance, in the data from Peru, IDV's culturally specific knowledge on the issue of gender inequality, and how it is addressed in their home communities, is recognized as valuable by the staff of partner organizations; partner organization staff expressed their intent on applying these approaches to the context in which they live in order to destabilize unequal power relations. However, the data from Senegal reveals that host communities do not just replace their own approaches with that of IDVs, rather in these cultural transactions, hosts maintain their own knowledge and tools, and add the experiences, knowledge and unique insights of IDVs to their own collection to generate new tools and "formulate new realities". These findings provide insights into the synergistic impact of collaborative models between IDVs and partner organizations. 
Adding to the synergistic benefits of collaboration, findings from Kenya show that intercultural interactions result in the expansion of the partner community's worldview and aid in the cultivation of hope for the future. The expansion of worldviews is also experienced by the IDVs who gain valuable culturally-specific information as a result of their interactions with partner organizations and communities. The transfer of cultural knowledge builds the capabilities of IDVs and partner organizations by equipping both with the knowledge and tools to address issues of inequality in the spaces in which they live and work. These exchanges also expand the worldviews of IDVs and partner organizations, inspiring both to dream of their future and themselves, aiding in the cultivations of spaces in which they can express their agency.

Cross-cultural exchanges between IDVs and partners provide rich opportunities for reciprocal learning and knowledge sharing. The evidence in this research echoes that of Lough and Oppenheim (2017) who find that relations of cultural exchange are reciprocal in nature and thus beneficial to both. In the exchanges of knowledge and experiences, IDVs become informed of cultural practices, particularly surrounding gender roles and norms through conversations with organizational staff and members of the community. Partner organization staff play a pivotal role in shaping the knowledge and understanding of IDVs by helping them develop their knowledge of the context, culture, history, and interpersonal and inter-institutional relationships. To further enhance the opportunities for expanded learning among IDVs, partner organizations suggested that IDVs spend more time at the start of their mandates developing an understanding of the nature of power relations in the spaces in which they are working.

Greater attention to improved training and orientation of IDVs to local cultural realities is believed to be a strategy that will better equip IDVs to be effective when addressing issues in the local contexts and enhance their performance throughout their mandates. This recommendation is supported by the research of Mclachlan and Binns (2019) who observe that the knowledge extracted from partner communities on values, traditions, and practices are essential in delivering successful grassroots development projects. The power and agency of hosts is expressed in the exchanges with IDVs as they aid in building the capabilities of IDVs by first identifying their knowledge and skill gaps and then curating the knowledge and tools for these volunteers to better steer the direction of their own capacity development.

Among the most significant contributions made by IDVs, networking and bridging relationships was seen as highly valuable. IDVs played an important role as liaisons between partner organizations and other development agencies, funders and institutions which were seen as constructive and valuable to the operations of partner organizations. Yet, this contribution must be considered in relation to the 
structural challenges that prevail. Persistent power imbalances between the Global North and Global South continue to privilege the voices of IDVs over local staff, thus perpetuating unequal relations (Perold et al. 2013; Lough/Oppenheim 2017). However, an analysis of the perspectives of partner organizations and communities on these relations of exchange brings nuance to these discussions. For instance, hosts reported that IDVs have a voice when they introduce topics and discuss "controversial" or foreign ideas with local staff. Their position as foreigners privileges their knowledge and voice with understanding and respect from the local staff, local agencies, and institutions. This gives IDVs an avenue to champion the priorities of the partner organization staff. Also, IDVs were able to use their privilege to call for the increased participation of women, and in advocating for equal participation of women in activities, as well as aiding in the inclusive policy making of partner organizations (Aked 2015). Thus, IDVs are seen by partner organization staff as a way to begin to deconstruct unequal power relations.

The power of host organizations and communities is not only cultivated through these relations of exchange with IDVs, it also exists and is expressed prior to and during these exchanges. The findings demonstrate that the partner organizations express their power through sharing their perspectives on what type of relationship has worked for them in building their capacity, the ways in which it has worked, as well as detailing how these relationships are cultivated, thus indirectly determining the terms of their relationship. For example, the data from Tanzania reveals that partnerships between IDVs and host organization staff are valued as they are constituted by collaborative practices in which both parties contribute equally, value each other's perspectives, and come to mutual understandings and unanimous decisions. The data illustrates that host organization staff value their own power and recognize that both parties bring valuable knowledge and skills to the table. Thus, in communicating their perspectives and preferences, partners are actively defining the terms of these relationships.

\section{Conclusion}

Through the lens of capabilities development, this paper examined the nature of transnational exchanges between IDVs and partner organization staff and communities in relation to capacity building. In this paper, capabilities development refers to the process of cultivating an environment in which people have the opportunities and freedoms to choose how to live (Sen 1999). With respect to the capacity building of partner organizations and communities, these partners worked collaboratively with IDVs to develop their capabilities, integral to the process of capacity building. Through the transfer of skills and knowledge, both technical and culturally specific, both IDVs and partner organization staff and communities become equipped with the tools to address the symptoms as well as the root causes 
of issues, and work towards building their respective capabilities. The combined focus on symptoms and root causes in development work was particularly important to GEWE policymaking and programming. In fact, partner organization staff revealed that gender inclusive policymaking and programming was championed by IDVs, who served as valuable resources to these organizations. Evidenced to have impactful voices in spaces in which partner organization staff and communities do not, IDVs utilize their privilege and take on the role of representing the interests of these staff and communities in matters of capacity building and capability development. However, partner organization staff exercise power in practices of capacity building with IDVs, as they define the terms of the relationships of exchange in sharing their perspective of the nature of relationship to be built and through which mechanisms they deem most effective in building capacity. Partnership and reciprocity, cultivated through a commitment to relationship building characterized the nature of the collaborative capacity building process between IDVs and partner organizations and communities.

\section{Bibliography}

Aked, Jody (2015): What's different about how volunteers work? Relationship building for wellbeing and change. In: IDS Bulletin, vol. 46, no. 5, pp. 29-42.

Ferguson, Lucy (2015): This is our gender person. The messy business of working as a gender expert in international development. In: International Feminist Journal of Politics, vol. 17, no.3, pp. 380-397.

Girgis, Mona (2007): The capacity building paradox. Using friendship to build capacity in the South. In: Development in Practice, vol. 17, no. 3, pp. 353-366.

Hawkes, Martine (2014): International volunteerism. Supports and barriers to capacity development outcomes. In: Third Sector Review, vol. 20, No. 1, pp. 1-11.

Impey, Kathy; Overton, John (2013): Developing Partnerships. The Assertion of Local Control of International Development Volunteers in South Africa. In: Community Development Journal, vol. 49, No. 1, pp. 111-128.

Kothari, Uma (2005): Authority and Expertise. The Professionalization Of International Development And The Ordering Of Dissent. In: Antipode, pp. 425-446.

Lough, Benjamin J.; Moore-McBridge, Amanda; Sherraden, Margaret S.; O'Hara, Kathleen (2011): Capacity building contributions of short-term international volunteers. In: Journal of Community Practice, vol. 19, pp. 120-137.

Lough, Benjamin J.; Oppenheim, Willy (2017): revisiting reciprocity in international volunteering. In: Progress in Development Studies, vol. 17, no. 3, pp. 197-213.

McLachlan, Sam; Binns, Tony (2019): Exploring Host Perspectives Towards Younger International Development Volunteers. In: Development in Practice, vol. 29, no. 1, pp. 65-79.

Nussbaum, Martha (2011): Creating Capabilities. The Human Development Approach. Cambridge.

Nyirenda, Denis (2018): Malawi: Mixed Results Form International Volunteers in Organizational Development and Capacity Building. In: Tiessen, Rebecca; Lough, Benjamin J.; 
Grantham, Kate (ed.): Insights on International Volunteering. Perspectives From the Global South. Germany, pp. 87-97.

OECD (2011): The enabling environment for capacity development. https://www.oecd.org/ dac/accountable-effective-institutions/48315248.pdf (30.6.2021).

Parpart, Jane L. (1995): Chapter 12: deconstructing the development expert: gender, development and the vulnerable. In: Marchand, Marianne H.; Parpart, Jane L. (ed.): Feminism/ Postmodernism/Development. London, pp. 221-243.

Perold, Helena; Graham, Lauren A.; Mazembo Mavungu, Eddy; Cronin, Karena; Muchemwa, Learnmore; Lough, Bejamin (2013): The colonial legacy of international voluntary service. In: Community Development Journal, vol. 48, pp. 179-96.

Roberts, Adrienne; Soederberg, Susanne (2012): Gender equality as smart economics? A critique of the 2012 World Development Report. In: Third World Quarterly, vol. 33, no. 5, pp. 949-968.

Sen, Amartya (1999): Development as Freedom. New York.

Tiessen, Rebecca; Lough J., Benjamin (2018): International volunteering capacity development. Volunteer partner organization experiences of mitigating factors for effective practice. In: Forum for Development Studies, pp. 1-21.

Wilson, Kalpana (2015): Towards a radical re-appropriation: gender, development and neoliberal feminism. In: Development and Change, vol. 46, no. 4, pp. 803-832. 